\title{
Jogos Sérios no Processo de Ensino e Aprendizagem de Interação Humano-Computador
}

\author{
André Barros de Sales ${ }^{1}$, Mateus Augusto Sousa e Silva ${ }^{1}$ \\ ${ }^{1}$ Faculdade UnB Gama (FGA) - Universidade de Brasília - Gama, DF - Brasil \\ andrebdes@unb.br, mateus.augusto@aluno.unb.br
}

\begin{abstract}
Aiming to assist in the choice of serious games to support the teaching and learning process of the Human-Computer Interaction (HCI) discipline, this article presents a Systematic Literature Review (SLR) with the Reverse Snowballing procedure of articles published in the ACM databases, Springer, Scopus and the Extended Proceedings of the Brazilian Symposium on Human Factors in Computer Systems. The result includes a comparison of the selected serious games; the RSL synthesis was subdivided into showing the features, non-functional requirements of the games and the user experience when using these games. The results reveal aspects that must be taken into account when choosing a serious game for HCI, in addition to noting the lack of research on the topic.
\end{abstract}

Resumo. Objetivando auxiliar nas escolhas de jogos sérios para apoiar o processo de ensino e aprendizagem da disciplina Interação Humano-Computador (IHC), este artigo apresenta uma Revisão Sistemática de Literatura (RSL) com o procedimento Snowballing Reverso de artigos publicados nas bases de dados da ACM, Springer, Scopus e dos Anais Estendidos do Simpósio Brasileiro de Fatores Humanos em Sistemas Computacionais. O resultado inclui comparativo dos jogos sérios selecionados; a sintese da RSL se subdividiu em mostrar as características, os requisitos não funcionais dos jogos e a experiência do usuário ao usar esses jogos. Os resultados revelam aspectos que devem ser levados em conta na escolha de um jogo sério para IHC, além de constatar a carência de pesquisas sobre o tema.

\section{Introdução}

A tecnologia da informação vive em constante processo de mudança e inovação. Os profissionais dessa área, como os engenheiros de software, devem ser capazes de desenvolver aplicações que atendam as expectativas dos usuários. Sendo assim, é necessário ter conhecimento e familiaridade com as técnicas, ferramentas e métodos que vêm da Engenharia de Software [Queiroz et al. 2017]. É imprescindível que os profissionais que desenvolvem software conheçam a área de Interação Humano-Computador (IHC). Na busca de melhorar a qualificação desses profissionais, diversas abordagens de ensino e ferramentas são utilizadas no processo de ensino e aprendizagem de IHC, como descritas em [Lima et al. 2019, Sales and Boscarioli 2020, Sales et al. 2020].

Este trabalho apresenta uma Revisão Sistemática de Literatura sobre o uso de Jogos Sérios (serious games) como apoio ao processo de ensino-aprendizagem em IHC. Foi realizado um planejamento com foco em mostrar as características, a experiência dos jogadores e os requisitos não funcionais desses jogos. A seguir temos a Seção 2, 
Metodologia; a Seção 3, Discussão dos Resultados; a Seção 4, Considerações Finais; e por fim as referências bibliográficas.

\section{Metodologia}

Em termos de objetivos, o estudo é de cunho descritivo; quanto à abordagem, é do tipo qualitativo [Gerhardt and Silveira 2009]; quanto aos procedimentos, é do tipo pesquisa bibliográfica através de Revisão Sistemática de Literatura (RSL). A RSL é uma metodologia de estudo secundário que promove um levantamento formal do estado da arte por meio de planejamento e execução criteriosos. A metodologia adotada para a realização desta RSL seguiu as diretrizes propostas por [Kitchenham 2004].

Segundo [Petersen et al. 2015], pode-se obter melhores resultados em uma pesquisa ao serem combinadas a string de busca com estratégias secundárias, como Snowballing. O Snowballing consiste em avaliar a lista de referências e citações de um conjunto de estudos primários relevantes para identificar outros estudos relevantes [Wohlin 2014]. Para completar a RSL, foi adicionado o procedimento Snowballing Reverso.

A RSL é desenvolvida em três fases: Planejamento, Condução e Publicação dos Resultados (Figura 1). Essas fases e atividades são descritas nas próximas seções.

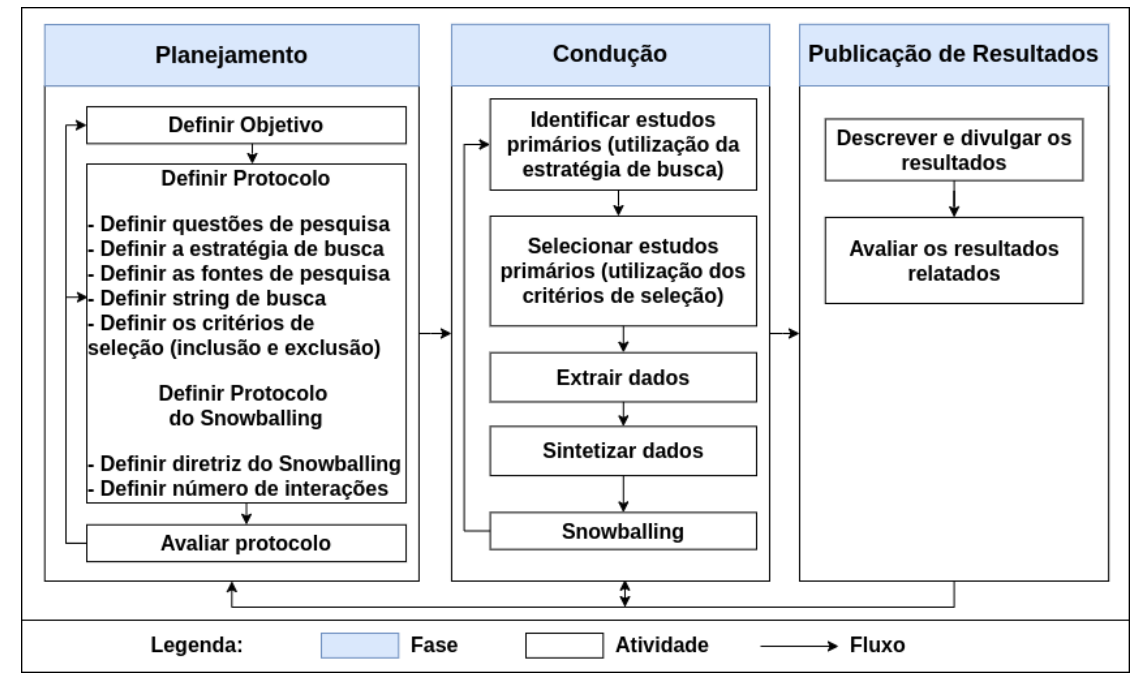

Figura 1. Fases e atividades da RSL - adaptado de [Felizardo et al. 2017].

\subsection{Planejamento}

O objetivo deste estudo é responder à seguinte questão de pesquisa: Quais as especificações utilizadas no design de jogos sérios aplicados no ensino de IHC? Para responder esta questão, foram feitas 3 outras Questões de Pesquisa: Quais as características desses jogos? Qual a Experiência do Usuário ao usar esses jogos? Quais os Requisitos Não Funcionais desses jogos?

A experiência do usuário envolve o modo como o uso de um produto afeta os sentimentos e as emoções do usuário ao usá-lo, olhá-lo, abri-lo ou fechá-lo [Rogers et al. 2013], conceito adotado nesta pesquisa. A experiência do usuário é um fator importante na análise de jogos educacionais. 
Os RNF podem ainda ser classificados de acordo com o que eles são: requisitos de desempenho, requisitos de manutenção, requisitos de segurança, requisitos de confiabilidade, requisitos de interoperabilidade ou um dos muitos outros tipos de requisitos de software. Segundo SWEBOK [Bourque et al. 2014], os RNF são os que atuam para restringir a solução, também conhecidos como requisitos restrições ou requisitos de qualidade. Os RNF influenciam a experiência do usuário, como por exemplo a facilidade na interação com a interface, oferecer feebacks e tempo de resposta.

A fim de responder essas questões de pesquisa, foram realizadas buscas de artigos científicos nas bases ACM, Springer, Scopus e nos Anais Estendidos do Simpósio Brasileiro de Fatores Humanos em Sistemas Computacionais disponíveis até 30/06/2020. Para a busca foi usada a string ("serious game"OR "gamification"OR "educational game") AND "hci course", mas nos Anais Estendidos do Simpósio Brasileiro de Fatores Humanos em Sistemas Computacionais os operadores "AND" e "OR" que estavam em maiúsculas na string de busca tiveram de ser trocados para minúsculas, ficando o seguinte: ("serious game"or "gamification"or "educational game") and "hci course".

Para inclusão e exclusão foram adotados os critérios mostrados na Tabela 1.

Tabela 1. Critérios de Exclusão e Inclusão

\begin{tabular}{|c|l|l|}
\hline Critérios & Identificador & Descrição \\
& E1 & $\begin{array}{l}\text { Capítulo de livros, pôsteres, workshops, estudos secundários, artigos } \\
\text { indisponíveis e incompletos }\end{array}$ \\
\hline \multirow{2}{*}{ Exclusão } & E2 & Artigos em outro idioma, além de Português e Inglês \\
\hline & E3 & Artigos que não abordam o uso de jogos sérios. \\
\hline & E4 & Artigos que não auxiliem o ensino e a aprendizagem em cursos de IHC. \\
\hline \multirow{2}{*}{ Inclusão } & E5 & Artigos Duplicados \\
\hline & I1 & Artigos que apresentem as características dos jogos \\
\hline & I3 & Artigos que apresentem Requisitos Não Funcionais (RNF) dos jogos \\
\hline
\end{tabular}

Para complementar a RSL, foi adicionado o procedimento Snowballing Reverso. O conjunto de estudos primários do Snowballing foi obtido ao final da atividade de seleção da RSL, no qual foram aplicadas 2 interações do Snowballing Reverso.

\subsection{Condução}

2.2.1 RLS: Com a busca nas bases foi possível identificar 22 estudos primários. A partir disso foi realizada a atividade de seleção dos artigos, dividida em 2 etapas.

Etapa 1: Primeiramente, foram aplicados os critérios de exclusão E1 e E2. Na $\mathrm{ACM}$, foram retornados 4 resultados. Na Springer, foram retornados 11 resultados. Na Scopus, foram retornados 3 resultados. Na base dos Anais Estendidos do Simpósio Brasileiro de Fatores Humanos em Sistemas Computacionais, foram retornados 4 resultados. Ao todo, resultaram 18 artigos.

Etapa 2: Em seguida, foram lidos os títulos e resumos dos artigos, em alguns casos foram lidos outras seções, buscando identificar e excluir artigos que não tratassem de jogos sérios nem de jogos educacionais (educational games), segundo o critério de exclusão E3 e também se o estudo não auxiliava o ensino e a aprendizagem em cursos de 
IHC, segundo o critério de exclusão E4. Foram identificados 2 artigos duplicados, que foram eliminados segundo o critério de exclusão E5, restando então 3 artigos.

Esses 3 artigos foram lidos na íntegra e aqui incluídos para a análise dos resultados, além de serem usados como conjunto de estudos primários para a execução das interações do Snowballing Reverso.

2.2.2 Snowballing Reverso: Com base no conjunto de estudos primários foram identificados como candidatos à inclusão aqueles artigos descritos como "Trabalhos Relacionados", que foram referenciados pelos artigos do conjunto de estudos primários. Estes foram identificados nas seções de Trabalhos Relacionados ou na Introdução do próprio artigo. Foram adotados os mesmos critérios de exclusão descritos nas etapas 1 e 2 para selecioná-los. No Snowballing houveram 2 interações, onde os artigos incluídos neste estudo na primeira interação serviram como conjunto de estudos primários para uma segunda interação do Snowballing.

Interação 1: Nesta primeira interação foram identificados 15 candidatos a inclusão. Destes, restaram 13 artigos da etapa 1. Da etapa 2, restaram 4 artigos, que foram acrescentados ao estudo.

Interação 2: Na segunda interação foram identificados 26 candidatos a inclusão. Destes, restaram 22 artigos da etapa 1 . Da etapa 2, restou apenas 1 artigo, que foi acrescentado ao estudo.

A Figura 2 ilustra o processo para obter os 8 artigos com base nas atividades de identificação e seleção dos estudos. Estes foram lidos por completo para executar a atividade de extração e síntese dos dados.

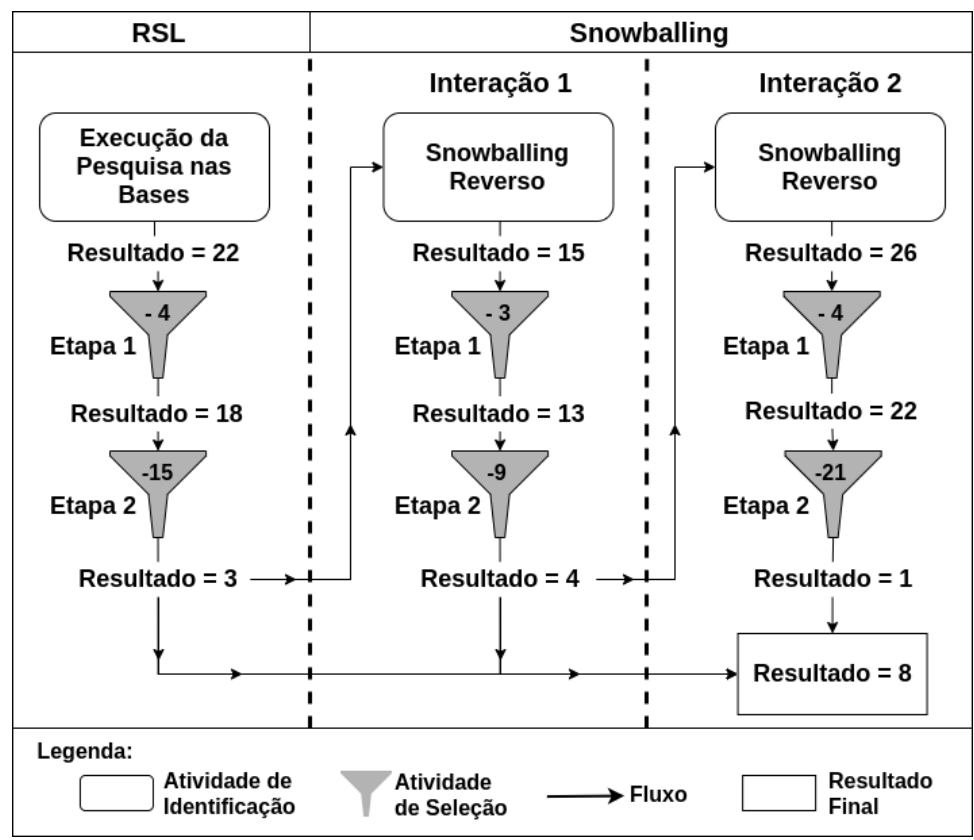

Figura 2. Processo de Identificação e Seleção

2.2.3 Extração e Síntese: Foram extraídos os dados dos artigos segundo os critérios de inclusão (I1, I2, I3). Os 8 trabalhos restantes passaram pela fase de extração e foram usados para responder às questões de pesquisa, através da leitura completa dos 
IX Congresso Brasileiro de Informática na Educação (CBIE 2020)

Anais do XXXI Simpósio Brasileiro de Informática na Educação (SBIE 2020)

trabalhos (Tabela 2).

Tabela 2. Artigos Selecionados x Critérios de Inclusão

\begin{tabular}{|c|c|c|c|c|c|}
\hline \multirow{2}{*}{ Atividade de Seleção } & \multirow{2}{*}{$N^{o}$ de Trabalhos } & \multirow{2}{*}{ Referências } & \multicolumn{3}{|c|}{ Critérios } \\
\hline & & & I1 & $\mathbf{I} 2$ & I3 \\
\hline \multirow{3}{*}{ RSL } & \multirow{3}{*}{3} & [Jucá et al. 2017] & $\mathrm{x}$ & - & $\mathrm{x}$ \\
\hline & & [de Souza Filho et al. 2019] & $\mathrm{x}$ & - & $\mathrm{x}$ \\
\hline & & [Darin et al. 2019] & $\mathrm{x}$ & - & - \\
\hline \multirow{4}{*}{ SnowBalling - Interação 1} & \multirow{4}{*}{4} & [Sommariva et al. 2011] & $\mathrm{x}$ & $\mathrm{x}$ & $\mathrm{x}$ \\
\hline & & [Ferreira et al. 2014a] & $\mathrm{x}$ & $\mathrm{x}$ & $\mathrm{x}$ \\
\hline & & [Ferreira et al. 2014b] & $\mathrm{x}$ & $\mathrm{x}$ & $\mathrm{x}$ \\
\hline & & [Queiroz et al. 2017] & $\mathrm{x}$ & $\mathrm{x}$ & $\mathrm{x}$ \\
\hline SnowBalling - Interação 2 & 1 & [Brito et al. 2016] & $\mathrm{x}$ & $\mathrm{x}$ & $\mathrm{x}$ \\
\hline
\end{tabular}

\section{Discussão dos Resultados}

A Tabela 3 exibe os 6 jogos apresentados nos 8 trabalhos selecionados. O primeiro jogo foi apresentado em 2011.

Tabela 3. Identificação dos Jogos

\begin{tabular}{|c|c|}
\hline Referências & Nome do Jogo \\
\hline [Sommariva et al. 2011] & UsabilityGame \\
\hline [Ferreira et al. 2014a] e [Ferreira et al. 2014b] & UsabiliCity \\
\hline [Brito et al. 2016] e [Queiroz et al. 2017] & MACteaching \\
\hline [Jucá et al. 2017] & G4H \\
\hline [de Souza Filho et al. 2019] & G4NHE \\
\hline [Darin et al. 2019] & Desafio de Design Goople (DDG) \\
\hline
\end{tabular}

Para responder às questões de pesquisa, essa análise sintetiza as descrições dos trabalhos lidos.

[Sommariva et al. 2011] apresentam o jogo sério UsabilityGame do gênero simulador e para um jogador: tem como cenário uma empresa de desenvolvimento de software fictícia (Booble Corporate) onde o jogador, no papel de engenheiro de usabilidade, participa de processos de desenvolvimento relacionados a design de interação.

[Ferreira et al. 2014a, Ferreira et al. 2014b] demonstram o jogo sério UsabiliCity. Por meio de analogias com problemas numa cidade, o estudante tem o papel de um inspetor (representado por um menino com uma lupa) que deve ajudar a identificar e solucionar os problemas de uma cidade (UsabiliCity) utilizando as dez heurísticas da técnica Avaliação Heurística [Nielsen 1994].

[Brito et al. 2016, Queiroz et al. 2017] apresentam o jogo sério MACteaching onde o estudante é inserido na história do jogo, na cidade Interacionópolis, onde são apresentados os conceitos da área por meio de analogias e personagens. A bruxa Ruptura insere várias rupturas de interação nas interfaces dos sistemas gerados dentro da cidade. 
O objetivo é identificar, para cada ruptura, qual a etiqueta do método que expressa o problema de comunicação na interface gráfica dos sistemas interativos. Para resolver a situação, através do Método de Avaliação de Comunicabilidade (MAC), o jogo disponibiliza ainda descrição das treze etiquetas do MAC, com interface intuitiva e amigável. Até o momento, a versão inicial do jogo é de nível iniciante e intermediário.

[Jucá et al. 2017] apresentam o jogo de cartas G4H (Game for Heuristic Evaluation) para aumentar o envolvimento de diferentes avaliadores num processo de avaliação baseado na Avaliação Heurística.

[de Souza Filho et al. 2019] apresentam o jogo de cartas G4NHE (Game for aNy Heuristic Evaluation), uma generalização do G4H como proposta para aumentar o envolvimento de diferentes avaliadores num processo de avaliação baseado na avaliação heurística aplicada às diferentes heurísticas de usabilidade.

[Darin et al. 2019] apresentam o jogo de cartas Desafio de Design Goople (DDG) para apoiar o ensino do design de interação e conceitos básicos de IHC.

Dos 6 jogos apresentados nos artigos, 3 são jogos de cartas, 2 jogos de aprendizagem por analogias que acontece resolvendo problemas de usabilidade numa cidade. O último é um simulador onde o jogador participa de processos de desenvolvimento de software relacionados a design de interação.

A seguir, a análise dos 6 jogos dos oito artigos selecionados para responder às questões de pesquisas elaboradas na fase de planejamento da RSL.

\subsection{Quais as características apresentadas por esses jogos?}

A Tabela 4 resume as características dos jogos sérios utilizados na disciplina de IHC dos artigos selecionados.

Tabela 4. Características dos Jogos

\begin{tabular}{|c|c|c|c|c|c|c|c|}
\hline \multicolumn{2}{|c|}{ Características } & DDG & G4NHE & $\mathrm{G} 4 \mathrm{H}$ & UsabiliCity & MACteaching & UsabilityGame \\
\hline \multicolumn{2}{|c|}{ Digital } & - & - & - & $\mathrm{x}$ & $\mathrm{x}$ & $\mathrm{x}$ \\
\hline \multicolumn{2}{|c|}{ Número de Jogadores } & 4 & {$[3,6]$} & {$[3,6]$} & 1 & 1 & 1 \\
\hline \multicolumn{2}{|c|}{ Duração Média de uma Partida } & - & - & - & $11 \mathrm{~min}$ & - & - \\
\hline \multicolumn{2}{|c|}{ Duração Máxima de uma Partida } & - & - & - & $27 \mathrm{~min}$ & - & - \\
\hline \multirow{4}{*}{$\begin{array}{c}\text { Gênero do } \\
\text { Jogo }\end{array}$} & Jogo de Analogias & - & - & - & $\mathrm{x}$ & $\mathrm{x}$ & - \\
\hline & Jogo de Carta & $\mathrm{x}$ & $\mathrm{x}$ & $\mathrm{x}$ & - & - & - \\
\hline & Jogo de Simulação & - & - & - & - & - & $\mathrm{x}$ \\
\hline & Jogo de Tabuleiro & $\mathrm{x}$ & - & - & - & - & - \\
\hline
\end{tabular}

Dos 6 jogos apresentados, três jogos de cartas (DDG, G4NHE e G4H) são jogos não digitais; o Jogo DDG é um jogo de cartas com tabuleiro que deve ser jogado com 4 jogadores; os jogos G4NHE e G4H podem ser jogados com 3 a 6 jogadores.

UsabiliCity, MACteaching e o UsabilityGame são os jogos sérios digitais para um jogador. O UsabiliCity e MACteaching são jogos para aprendizagem por analogias, e o UsabilityGame simula um processo de design de interação. Sobre o tempo de duração da partida do jogo, o tempo máximo da partida observado foi no UsabiliCity: 27 minutos, que o habilita para uso durante uma aula de IHC. 
Na Tabela 5, os conteúdos de IHC e o conhecimento necessário que o aluno deve ter, em IHC, para jogar cada jogo. Esse nível de conhecimento, conforme descrito nos artigos, foram divididos em: iniciante, intermediário e avançado.

Tabela 5. Conteúdo de IHC utilizados nos jogos.

\begin{tabular}{|c|c|c|c|c|c|c|c|}
\hline \multicolumn{2}{|c|}{ Características } & DDG & G4NHE & G4H & UsabiliCity & MACteaching & UsabilityGame \\
\hline \multirow{5}{*}{ Conteúdo } & $\begin{array}{l}\text { Princípios de Usabilidade } \\
\text { [Nielsen 94] }\end{array}$ & - & - & - & $\mathrm{x}$ & - & - \\
\hline & Coleta e Análise de Requisitos & $\mathrm{x}$ & - & - & - & - & $\mathrm{x}$ \\
\hline & Design & $\mathrm{x}$ & - & - & - & - & $\mathrm{x}$ \\
\hline & Prototipação & $\mathrm{x}$ & - & - & - & - & $\mathrm{x}$ \\
\hline & Avaliação & $\mathrm{x}$ & $\mathrm{x}$ & $\mathrm{x}$ & - & $\mathrm{x}$ & $\mathrm{x}$ \\
\hline \multirow{3}{*}{$\begin{array}{l}\text { Nível de } \\
\text { Conhecimento de } \\
\text { IHC dos } \\
\text { Jogadores }\end{array}$} & Iniciante & $\mathrm{x}$ & - & - & $\mathrm{x}$ & $\mathrm{x}$ & - \\
\hline & Intermediário & - & - & - & - & $\mathrm{x}$ & $\mathrm{x}$ \\
\hline & Avançado & - & $\mathrm{x}$ & $\mathrm{x}$ & - & - & $\mathrm{x}$ \\
\hline
\end{tabular}

O DDG é um jogo não digital de cartas com tabuleiro para iniciantes e pode ser usado no apoio ao processo de ensino e aprendizagem nos diversos momentos da disciplina de IHC.

O UsabiliCity é um jogo digital para iniciantes e pode ser usado no apoio ao processo de ensino e aprendizagem do conteúdo dos Princípios de Usabilidade [Nielsen 1994] na disciplina de IHC.

O MACteaching é um jogo digital que pode ser utilizado no processo de ensino e aprendizagem no momento em que o Método de Avaliação de Comunicabilidade (MAC) for ministrado, pois se destina a jogadores de nível iniciante e intermediário.

O G4NHE e o G4H se destinam a jogadores com nível de conhecimento dos conteúdos de IHC mais avançado.

\subsection{Qual a Experiência do Usuário ao usar o jogo?}

Na Tabela 6, a experiência do usuário identificados nos trabalhos lidos.

Tabela 6. Experiência do Usuário

\begin{tabular}{|c|c|c|c|c|c|}
\hline \multicolumn{2}{|c|}{ Aspectos da Experiência do Usuário } & \multirow{2}{*}{ G4H } & \multirow{2}{*}{$\begin{array}{c}\text { Usabilicity } \\
-\end{array}$} & \multirow{2}{*}{$\begin{array}{c}\text { MACteaching } \\
-\end{array}$} & \multirow{2}{*}{$\begin{array}{c}\text { UsabilityGame } \\
\text { X }\end{array}$} \\
\hline \multirow{6}{*}{ Desejáveis } & Apoia a Criatividade & & & & \\
\hline & Motivador & $\mathrm{x}$ & $\mathrm{x}$ & - & - \\
\hline & Atraente & $\mathrm{x}$ & $\mathrm{x}$ & $\mathrm{x}$ & - \\
\hline & Satisfatório & $\mathrm{x}$ & $\mathrm{x}$ & $\mathrm{x}$ & - \\
\hline & Divertido & $\mathrm{x}$ & $\mathrm{x}$ & - & $\mathrm{x}$ \\
\hline & Desafiador & - & $\mathrm{x}$ & - & - \\
\hline \multirow{3}{*}{ Indesejáveis } & Forçosamente Bonito (Cutesy) & - & $\mathrm{x}$ & $\mathrm{x}$ & - \\
\hline & Sentimento de Apreensão & $\mathrm{x}$ & - & - & - \\
\hline & Sentimento de desapontamento & - & $\mathrm{x}$ & $\mathrm{x}$ & - \\
\hline
\end{tabular}

[Sommariva et al. 2011] entendem que as fases do UsabilityGame dão liberdade para a criatividade do jogador ao fazer uma análise particular dos requisitos na primeira 
fase, em elaborar seu próprio protótipo na segunda fase e fazer avaliação heurística na terceira fase.

Segundo [Ferreira et al. 2014a, Ferreira et al. 2014b], os participantes da pesquisa relatam que o Usabilicity é um jogo que motiva ao aprendizado: $79 \%$ dos participantes se divertiram com o jogo e $66 \%$ consideraram as tarefas do jogo adequadamente desafiadoras. Sobre o item Forçosamente Bonito (Cutesy), alguns alunos relataram que a tela continha muita informação e a fonte do texto e/ou cores não eram agradáveis. Em relação aos aspectos indesejáveis da experiência do usuário, $48 \%$ deles ficaram desapontados com a interrupção do fim do jogo.

No jogo MACteaching, [Brito et al. 2016] acreditam que a disputa com outros jogadores pode torná-lo mais atrativo. Em relação aos aspectos indesejáveis da experiência do usuário, sobre o item Forçosamente Bonito (Cutesy), alguns participantes relataram dificuldade em ler legendas do jogo; $83 \%$ dos participantes ficaram desapontados com a interrupção do fim do jogo (gostariam de jogar mais). Após a avaliação do MACteaching foram feitas melhorias, embora essa nova versão ainda não tenha sido avaliada.

No jogo de cartas G4H, [Jucá et al. 2017] consideram que pessoas muito competitivas podem criar uma tensão que impeça outros jogadores de fazer comentários, o que não foi problema no estudo, porque os participantes da pesquisa eram colegas e levaram os comentários na brincadeira. Os autores ressaltam que esse problema poderia ocorrer em todos os métodos colaborativos, não apenas neste jogo.

No jogo de cartas G4NHE, [de Souza Filho et al. 2019] esperam que, após a avaliação, o jogo possa gerar os mesmos sentimentos de engajamento (atratividade), de ludicidade no ensino (divertido) e satisfação produzidos pelo $\mathrm{G} 4 \mathrm{H}$.

\subsection{Quais os Requisitos Não Funcionais desses jogos?}

Os requisitos identificados nos trabalhos lidos foram de Usabilidade e Portabilidade. Na Tabela 7, os requisitos não funcionais de usabilidade identificados nos trabalhos lidos. Sobre os requisitos não funcionais de portabilidade, o UsabiliCity e o UsabilityGame podem ser acessados por um navegador de internet, enquanto o MACteaching é um aplicativo para o sistema operacional Android.

Tabela 7. Requisitos Não Funcionais de Usabilidade

\begin{tabular}{|l|c|c|c|}
\hline Requisitos Não Funcionais & Usabilicity & MACteaching & UsabilityGame \\
\hline Promove facilidade na interação do jogador & $\mathrm{X}$ & $\mathrm{X}$ & $\mathrm{x}$ \\
\hline Permite executar as atividades do jogo em tempo adequado & $\mathrm{X}$ & $\mathrm{X}$ & - \\
\hline Aborda os conteúdos de forma a facilitar a aprendizagem. & $\mathrm{X}$ & $\mathrm{X}$ & $\mathrm{x}$ \\
\hline Oferece feedbacks & $\mathrm{X}$ & $\mathrm{X}$ & $\mathrm{x}$ \\
\hline Possui Narrativa & $\mathrm{X}$ & $\mathrm{X}$ & $\mathrm{x}$ \\
\hline Oferece pontos e recompensas & - & $\mathrm{X}$ & $\mathrm{x}$ \\
\hline Apresenta ranking dos jogadores & - & $\mathrm{X}$ & $\mathrm{x}$ \\
\hline
\end{tabular}

\section{Considerações Finais}

Este artigo apresentou uma Revisão Sistemática de Literatura sobre o uso de Jogos Sérios (serious games) como apoio ao processo de ensino e aprendizagem dos conteúdos da 
disciplina de Interação Humano-Computador, com foco em sintetizar e apresentar as características, os requisitos não funcionais e a experiência do usuário (jogador) ao usar esses jogos dos trabalhos compulsados.

Através dos artigos selecionados, foi possível identificar seis jogos sérios que utilizam conteúdos de Interação Humano-Computador: três são de cartas e não digitais; os outros três jogos são digitais e para um jogador; dois deles apresentam algum conteúdo de IHC através de analogias e o outro por simulação. Apenas um jogo digital, UsabiliCity, cita o tempo médio e máximo da partida, o que lhe permite ser utilizado durante uma aula de IHC.

O presente estudo permitiu conhecer uma síntese de jogos sérios que podem ser utilizados no processo de ensino e aprendizagem de Interação Humano-Computador. A análise dos jogos descritos evidenciou pontos importantes como: tipo do jogo, gênero, tempo médio da partida, número dos jogadores, grau de conhecimento dos jogadores em IHC necessários para jogar uma partida, qual conteúdo de IHC é apresentado no jogo, as características, requisitos não funcionais das interfaces e experiência dos usuários (jogadores) ao usar os jogos.

Encontra-se em andamento a avaliação do uso dos jogos digitais para identificar outros aspectos dos jogos selecionados, entre eles requisitos funcionais e os aspectos de gamificação. Como trabalhos futuros, aplicar essa RSL em anais de eventos voltados para ensino ou de informática na educação. Para apoiar o processo de ensino e aprendizagem de IHC, pretende-se desenvolver um jogo que contemple os diversos conteúdos da disciplina de IHC por fases do jogo e para os níveis iniciante e intermediário de conhecimento da disciplina.

\section{Referências}

Bourque, P., Fairley, R. E., et al. (2014). Guide to the Software Engineering Body of Knowledge (SWEBOK $(R))$ : Version 3.0. IEEE Computer Society Press.

Brito, R., Fernandes, M., Queiroz, W., Souza, B., Bonifácio, B., and Fernandes, P. (2016). Macteaching: Utilizando um jogo para apoio ao ensino do método de avaliação de comunicabilidade. Brazilian Symposium on Computers in Education (Simpósio Brasileiro de Informática na Educação - SBIE), 27(1):796.

Darin, T., Rocha, F., Motta, D., and Angelo, P. V. (2019). Desafio de design goople: Um jogo de cartas para apoio ao ensino do design de interação e conceitos básicos de interação humano-computador. In Anais Estendidos do XVIII Simpósio Brasileiro sobre Fatores Humanos em Sistemas Computacionais, pages 100-105, Porto Alegre, RS, Brasil. SBC.

de Souza Filho, J., Monteiro, I., and Jucá, P. (2019). Game for any heuristic evaluation ( $\mathrm{g} 4 \mathrm{nhe}$ ): a generalization of the $\mathrm{g} 4 \mathrm{~h}$ gamification considering different sets of usability heuristics. 18:489-505.

Felizardo, K. R., Nakagawa, E. Y., Fabbri, S. C. P. F., and Ferrari, F. C. (2017). Revisão Sistemática da Literatura em Engenharia de Software: Teoria e Prática. Elsevier Brasil. 
IX Congresso Brasileiro de Informática na Educação (CBIE 2020)

Anais do XXXI Simpósio Brasileiro de Informática na Educação (SBIE 2020)

Ferreira, B., Rivero, L., Lopes, A., Marques, A. B., and Conte, T. (2014a). Apoiando o ensino de qualidade de software: Um serious game para o ensino de usabilidade. VII Fórum de Educação em Engenharia de Software (FEES 2014), pages 12-21.

Ferreira, B. M., Rivero, L., Lopes, A., Marques, A. B., and Conte, T. (2014b). Usabilicity: Um jogo de apoio ao ensino de propriedades de usabilidade de software através de analogias. Brazilian Symposium on Computers in Education (Simpósio Brasileiro de Informática na Educação - SBIE), 25(1):1273.

Gerhardt, T. E. and Silveira, D. T. (2009). Métodos de pesquisa. Editora da UFRGS, Porto Alegre,Brazil, 1st edition.

Jucá, P. M., Monteiro, I. T., and de Souza Filho, J. C. (2017). Game for heuristic evaluation $(\mathrm{g} 4 \mathrm{~h})$ : a serious game for collaborative evaluation of systems. In International Conference on Human-Computer Interaction, pages 341-352. Springer.

Kitchenham, B. (2004). Procedures for performing systematic reviews. Keele, UK, Keele University, 33(2004):1-26.

Lima, J., Júnior, M. A., Moya, A., Almeida, R., amd Maria Lencastre, P. A., Fagundes, R., and Alencar, F. (2019). As metodologias ativas e o ensino em engenharia de software: uma revisão sistemática da literatura. Anais do Workshop de Informática na Escola, 25(1):1014.

Nielsen, J. (1994). Usability Engineering. Morgan Kaufmann.

Petersen, K., Vakkalanka, S., and Kuzniarz, L. (2015). Guidelines for conducting systematic mapping studies in software engineering: An update. Information and Software Technology, 64:1-18.

Queiroz, W., Beltrão, R., Fernandes, M., Bonifácio, B., and Fernandes, P. (2017). Macteaching: Uma abordagem para enriquecer o ensino do método de avaliação de comunicabilidade. Anais dos Workshops do Congresso Brasileiro de Informática na Educação, 6(1):138.

Rogers, Y., Sharp, H., and Preece, J. (2013). Design de Interação: Além da Interação Homem-Computador. Bookman Editora.

Sales, A. B. d. and Boscarioli, C. (2020). Uso de Tecnologias Digitais Sociais no Processo Colaborativo de Ensino e Aprendizagem. RISTI - Revista Ibérica de Sistemas e Tecnologias de Informação, pages $82-98$.

Sales, A. B. d., Serrano, M., and Serrano, M. (2020). Aprendizagem Baseada em Projetos na Disciplina de Interação Humano-Computador. RISTI - Revista Ibérica de Sistemas e Tecnologias de Informação, pages 49 - 64.

Sommariva, L., Benitti, F. B. V., and Dalcin, F. S. (2011). Usabilitygame: Jogo simulador para apoio ao ensino de usabilidade. IHC+CLIHC '11, page 61-65. Brazilian Computer Society.

Wohlin, C. (2014). Guidelines for snowballing in systematic literature studies and a replication in software engineering. In Proceedings of the 18th International Conference on Evaluation and Assessment in Software Engineering, EASE '14, New York, NY, USA. Association for Computing Machinery. 